\title{
Rede Combinatória de Modelos Não Lineares - Parte I: Métodos e Aplicação em Identificação Multiobjetivo de Sistemas *
}

\author{
Luan Pascoal da Costa Andrade* \\ Samir Angelo Milani Martins* \\ * GCOM - Grupo de Controle e Modelagem \\ Universidade Federal de São João del Rei \\ Praça Frei Orlando, 170 Centro. São João del Rei, MG, Brasil. \\ (e-mail:luan_pascoal13@hotmail.commatins@ufsj.edu.br)
}

\begin{abstract}
:
This work is an part of a series of two articles and proposes a combinatorial network of nonlinear models. The method proposes an analytical form of parallel model combining and an algorithm for calculating weights for each model is provided. The method is applied from submodels pertaining to the Pareto-Set obtained through a multiobjective procedure for selecting nonlinear structures. The composite model obtained better performance than the submodels used in the validation indexes analyzed, reaching a reduction of $65 \%$ in the NRMSE index, $95 \%$ in the MSE index of the static curve, $87 \%$ in the energy of the vector of residues and a reduction of $21 \%$ in the autocorrelation energy of the vector of residues.

Resumo: Este trabalho é parte integrante de uma série de dois artigos e propõe uma rede combinatória de modelos não lineares. O método traz uma forma analítica de combinar modelos paralelamente e um algoritmo para o cálculo dos pesos que ponderam os modelos é fornecido. A técnica é aplicada a partir de submodelos pertencentes ao conjunto Pareto-Ótimo obtidos através de um procedimento multiobjetivo de seleção de estruturas de modelos não lineares. $\mathrm{O}$ modelo composto obteve melhor desempenho que os submodelos utilizados em sua obtenção nos índices de validação analisados, obtendo uma redução de $65 \%$ no índice NRMSE, $95 \%$ no índice MSE da curva estática, $87 \%$ na energia do vetor de resíduos e uma redução de $21 \%$ na energia da autocorrelação do vetor de resíduos.
\end{abstract}

Keywords: Systems Identification; Combinatorial Network of Models ; Multiobjective Systems Identification.

Palavras-chaves: Identificação de Sistemas; Rede Combinatória de Modelos; Identificação Multiobjetivo de Sistemas.

\section{INTRODUÇÃO}

$\mathrm{Na}$ identificação de sistemas, nem sempre é possível se obter bons modelos, seja por dificuldades em coletar dados, mal condicionamento numérico ou por alta complexidade do sistema. Uma alternativa é a inserção de informação a priori, podendo ser de origem estática, localização dos pontos fixos e outros tipos de conhecimento prévio sobre o sistema. Uma técnica utilizada para tal é a identificação multiobjetivo na qual ao selecionar a estrutura ou ajustar os parâmetros do modelo utiliza-se técnicas que minimizam mais de uma função objetivo, denominadas otimizações multiobjetivo.

Um processo de identificação multiobjetivo resulta em uma família de modelos que minimizam os múltiplos objetivos e que estão no limiar entre estes. Quando estes objetivos são contrastantes, melhorar o desempenho em um objetivo

\footnotetext{
* O presente trabalho foi realizado com apoio da Coordenação de Aperfeiçoamento de Pessoal de Nível Superior - Brasil (CAPES) Código de Financiamento 001.
}

reduz o desempenho em outro. Este conjunto de modelos é denominado Pareto-Ótimo ou Soluções Eficientes. Diversas técnicas para encontrar este conjunto podem ser vistas na literatura, Johansen (2000) introduz uma metodologia para a identificação multiobjetivo de modelos FIR. Barbosa (2010) apresenta um procedimento bi-objetivo que auxilia na seleção de estruturas de modelos NARX polinomiais. Nepomuceno et al. (2003) utilizam o conhecimento a priori das localizações dos pontos fixos na identificação de sistemas não lineares em uma abordagem multiobjetivo que é posteriormente estendida para um número arbitrário de objetivos em (Nepomuceno et al., 2007). Em (Martins et al., 2013) é proposto o Multiobjective Error Reduction Ratio, um método multiobjetivo para a detecção da estrutura de modelos NARMAX polinomiais. Aplicações da identificação multiobjetivo também podem ser encontradas na literatura como a detecção de danos em pontes e estruturas (Ok et al., 2018), identificação de modelos químicos e fotoquímicos (Beykal et al., 2018) (Vignoni et al., 2018), problemas de visão computacional (Gao et al., 2018) e (Chevtchenko et al., 2018) entre outros. 
A identificação multiobjetivo de sistemas não consiste a apenas encontrar o conjunto Pareto-Ótimo mas também escolher dentro deste conjunto qual modelo utilizar, sendo o estágio de decisão uma parte crucial de qualquer otimização multiobjetivo. Diversos trabalhos da literatura endereçam a atenção para o problema de decisão, Aguirre et al. (2006) apresenta um método de validação de modelos não lineares que é utilizado em (Barbosa, 2010) como critério decisor em uma metodologia de Identificação Multiobjetivo, Barroso et al. (2007) propõe um critério de decisão baseado na mínima correlação entre o erro de simulação livre e a saída do modelo, a decisão multiobjetivo é utilizada no problema de alocação de recursos em (Ramalho et al., 2019). Estes procedimentos tem por objetivo selecionar apenas um modelo dentro do conjunto Pareto-Ótimo que melhor se adéque às necessidades segundo algum critério.

Este trabalho propõe uma rede combinatória de modelos não lineares que combina modelos de forma paralela. A metodologia é aplicada a modelos de baixo desempenho pertencentes ao conjunto Pareto-Ótimo, resultando em um modelo com maior desempenho. Assim bons modelos podem ser obtidos a partir de modelos com baixa performance. As Soluções Eficientes foram obtidas através do Multiobjective Error Reduction Ratio disponível em (Martins et al., 2013).

O procedimento ataca o problema de decisão fornecendo um único modelo homogêneo com melhor desempenho que os modelos utilizados em sua obtenção. O procedimento também vai além do problema de decisão uma vez que mais de um modelo pertencente ao conjunto Pareto-Ótimo é utilizado de forma simultânea. A técnica pode também ser utilizada fora do contexto da identificação multiobjetivo como pode ser visto em Rede Combinatória de Modelos Não Lineares - Parte II: Aplicação em Computação Aritmética.

O restante do trabalho é dividido da seguinte forma, a Seção 2 traz alguns conceitos preliminares, a Seção 3 apresenta a metodologia utilizada, na seção 4 são mostrados os resultados e a Seção 5 traz as conclusões e perspectivas de trabalhos futuros.

\section{CONCEITOS PRELIMINARES}

Esta Seção apresenta conceitos teóricos necessários ao entendimento deste trabalho.

\subsection{Modelos NARMAX polinomiais}

Os modelos NARMAX polinomiais são representações autorregressivas compostas por funções polinomiais das entradas e saídas passadas, e podem ser escritas como em (1):

$$
\begin{aligned}
y_{k}= & F^{\ell}\left[y_{k-1}, \ldots, y_{k-n_{y}}, u_{k-1}, \ldots\right. \\
& \left.u_{k-n_{u}}, e_{k-1}, \ldots, e_{k-n_{e}}\right]+e_{k}
\end{aligned}
$$

em que $y_{k}, u_{k}$ e $e_{k}$ são a saída, a entrada e a incerteza respectivamente, e $n_{y} n_{u}$ e $n_{e}$ os atrasos máximos correspondentes (Leontaritis e Billings, 1985a,b; Chen e Billings, 1989). $F^{\ell}[\cdot]$ é uma função polinomial de grau $\ell$.

\subsection{Critérios de Validação}

Raiz do Erro Quadrático Médio Normalizado O NRMSE é um índice utilizado para avaliar o desempenho dinâmico de modelos matemáticos que pode ser calculado como em (2) (Aguirre, 2015):

$$
\mathrm{NRMSE}=\frac{\sqrt{\sum_{k=1}^{N}\left(y_{k}-\hat{y}_{k}\right)^{2}}}{\sqrt{\sum_{k=1}^{N}\left(y_{k}-\bar{y}_{k}\right)^{2}}} .
$$

em que $\hat{y}_{k}$ é a saída do modelo, $y_{k}$ a saída real do sistema e $\overline{y_{k}}$ sua média em $N$ amostras.

Erro Quadrático Médio O Erro Quadrático Médio ou MSE (do inglês Mean Squared Error) é um índice que calcula a média dos erros quadráticos entre valores de uma predição e os valores reais a serem preditos, e pode ser calculado como em (3) (Aguirre, 2015):

$$
\mathrm{MSE}=\frac{1}{N} \sum_{k=1}^{N}\left(y_{k}-\hat{y}_{k}\right)^{2} .
$$

Em que $y_{k}$ é a saída real do sistema, $\hat{y}_{k}$ o valor da predição e $N$ o número de amostras utilizadas.

Análise de Resíduos $\mathrm{Na}$ análise de resíduos é verificado se os erros cometidos pelo modelo possuem características aleatórias e se são correlacionados com a entrada. Para tal é realizado o cômputo das funções de correlação cruzada entre o vetor de resíduos e a entrada (4) e de auto correlação do vetor de resíduos (5).

$$
\begin{aligned}
& r_{u \xi}(k)=\lim _{N \rightarrow \infty} \frac{1}{2 N+1} \sum_{i=-n}^{n} u(i) \xi(i+k) \\
& r_{\xi \xi}(k)=\lim _{N \rightarrow \infty} \frac{1}{2 N+1} \sum_{i=-n}^{n} \xi(i) \xi(i+k)
\end{aligned}
$$

em que $\xi$ é o vetor de resíduos, $u$ é o vetor de entrada, $k$ indica o tempo discreto e $N$ o tamanho da série temporal.

\subsection{Multiobjective Error Reduction Ratio}

O Multiobjective Error Reduction Ratio (MERR) é uma técnica multiobjetivo de seleção de estruturas de modelos NARMAX polinomiais que pode ser vista em (Martins et al., 2013). Se trata de uma extensão do já conhecido Error Reduction Ratio técnica capaz de ordenar regressores por relevância no que diz respeito a representatividade do sistema.

A técnica resulta em um conjunto Pareto-Ótimo ordenando os regressores candidatos por sua relevância na representação do sistema, ponderando a importância de cada conjunto de informações auxiliares utilizada, aqui chamada de informações afins. O MERR pode ser calculado por (6).

$$
M E R R_{i}=g_{i}^{2} \sum_{j=1}^{m} \omega_{j} \frac{\left\langle\Omega_{i, j}, \Omega_{i, j}\right\rangle}{\left\langle v_{j}, v_{j}\right\rangle}
$$

Em que $\langle.,$.$\rangle é o simbolo de produto interno, \Omega_{i, j}$ é o i-ésimo regressor ortogonal associado ao j-ésimo par de 
informações afins, $g_{i}$ o seu parâmetro, $\omega_{j}$ é o peso dado para o j-ésimo par de informações afins e $v_{j}$ é um vetor de informações sobre o sistema podendo conter dados estáticos, dinâmicos, de localização de pontos fixos entre outros.

A técnica é capaz de selecionar estruturas de modelos polinomiais que são melhores em uma característica do que outra, por exemplo um modelo com um bom comportamento dinâmico mas que deixa a desejar em características estáticas, ou um modelo que tem uma boa descrição dos pontos fixos do sistema mas perde em características dinâmicas.

\section{METODOLOGIA}

O Sistema piloto utilizado é puramente simulado sendo um modelo NARX polinomial fornecido em (Piroddi e Spinelli, 2003) e representado em (7). O sinal de entrada utilizado foi um ruído Gaussiano branco, com média nula e variância unitária. Foram utilizadas um total de 630 amostras, sendo 500 amostras utilizadas para identificação e 130 utilizadas para a validação.

$$
\begin{aligned}
y(k)= & 0,5 y(k-1)+0.8 u(k-2)+ \\
& u(k-1)^{2}-0.05 y(k-2)^{2}+0.5
\end{aligned}
$$

O sistema (7) foi identificado utilizando o MERR (Multiobjective Error Reduction Ratio) tendo como objetivos a redução dos erros quadráticos estático e dinâmico e utilizando para tal os seguintes pesos: $\omega_{d}=[0,1 ; 0,35] \mathrm{e}$ consequentemente $\omega_{s}=[0,9 ; 0,65]$. Os modelos utilizados podem ser vistos em (8) e (9). Estes modelos foram escolhidos por possuírem baixo desempenho e não representarem bem o comportamento do sistema, assim melhorias devem ser realizadas para que os modelos possam ser utilizados na representação do sistema.

$$
\begin{gathered}
y_{1}(k)=0.5821 y_{1}(k-1)+0.8184 u(k-2)+ \\
0.8348+0.9459 u(k-1)^{2}-0.3209 y_{1}(k-2) \\
-0.0292 y_{1}(k-2) y_{1}(k-1) \\
y_{2}(k)=0.7019 y_{2}(k-1)+0.8018 u(k-2) \\
-0.0695 y_{2}(k-2) y_{2}(k-1)+0.9786 u(k-1)^{2} \\
+0.0042 u(k-1) y_{2}(k-1) \\
-0.0812 u(k-1) y_{2}(k-2)
\end{gathered}
$$

O procedimento proposto consiste em combinar modelos pertencentes ao conjunto Pareto-Ótimo de forma paralela como pode ser visto na Figura 1 . O modelo composto $y^{*}$ é então uma soma ponderada dos modelos $y_{1}$ e $y_{2}$ pelos pesos $\mu_{1}$ e $\mu_{2}$.

Para a aplicação da metodologia o problema deve estar bem condicionado como na Figura 2, ou seja, a resposta real do sistema deve estar contida na região que compreende as respostas dos dois modelos utilizados na combinação. O bom condicionamento do problema é garantido testando a condição apresentada em (10). Caso a condição não seja satisfeita o problema pode ser condicionado

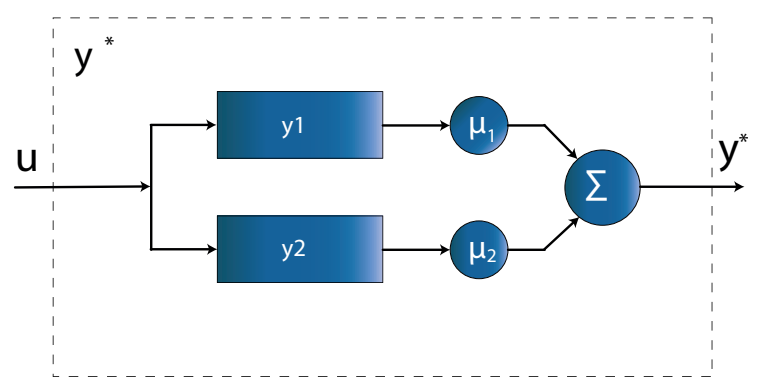

Figura 1. Rede Combinatória de Modelos Não Lineares, sendo $y^{*}$ o modelo composto, $y_{1}$ e $y_{2}$ os submodelos utilizados e $\mu_{1}$ e $\mu_{2}$ seus respectivos pesos. $u$ é a entrada.

calculando as grandezas apresentadas em (11) e (12) e realizando o restante do procedimento utilizando os modelos transformados $y_{1}^{*}(13)$ e $y_{2}^{*}(14)$ que garantem a condição (10).

$$
\begin{gathered}
y_{1_{\min }} \geq y_{\max } \geq y_{\min } \geq y_{2_{\max }} \\
c_{1}=y_{\max }+y_{1_{\min }} \\
c_{2}=y_{\min }+y_{2 \max } \\
y_{1}^{*}(k)=y_{1}(k)+c_{1} \\
y_{2}^{*}(k)=y_{2}(k)-c_{2}
\end{gathered}
$$

A resposta do modelo combinado visto na Figura 1 é definida em (15). Ao variar os pesos entre 0 e 1, assumindo $\mu_{1}+\mu_{2}=1$ a resposta do modelo composto da Figura 1 $y^{*}$ se desloca na região compreendida pelas respostas dos modelos $y_{1}$ e $y_{2}$. Sendo $\mu_{2}=1-\mu_{1}$ a resposta do modelo combinado pode ser representada também como em (16).

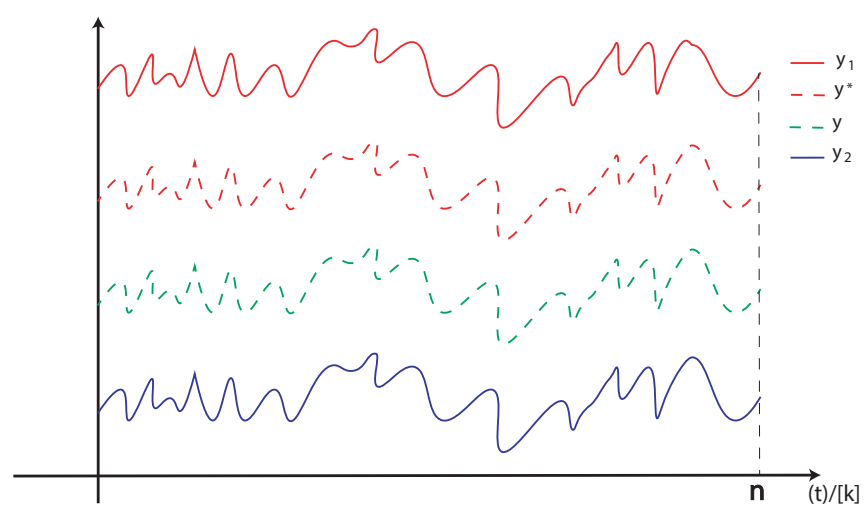

Figura 2. Saídas de modelos fictícios devido a entrada $u$. A resposta real do sistema $y$ deve estar compreendida entre a resposta dos modelos $y_{1}$ e $y_{2}$.

$$
\begin{gathered}
y^{*}=\mu_{1} y_{1}+\mu_{2} y_{2} . \\
y^{*}=\mu_{1} y_{1}+\left(1-\mu_{1}\right) y_{2} .
\end{gathered}
$$

Deseja-se que a saída do modelo composto seja a mais aproximada possível da saída real do sistema, ou seja $y^{*} \approx y$. Levando essa informação em (16) e abandonando o subscrito 1 em $\mu_{1}$ : 

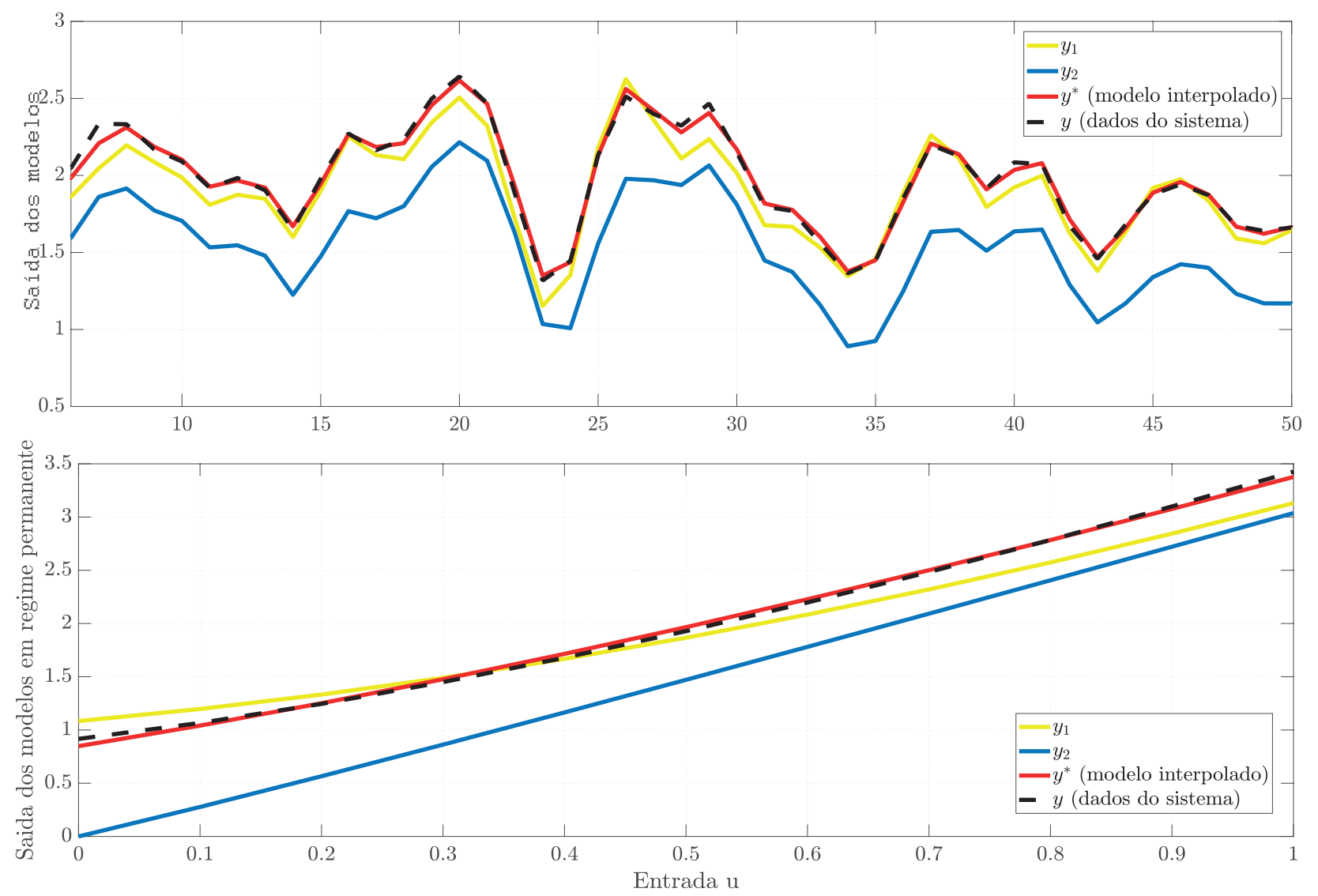

Figura 3. Acima: Simulação dos modelos $y_{1}$ e $y_{2}$ juntamente com o modelo combinado $y^{*}$ e os dados do sistema $y$; Abaixo: Curvas estáticas para os modelos $y_{1} \mathrm{e} y_{2}, y^{*}$ e para o sistema $y$
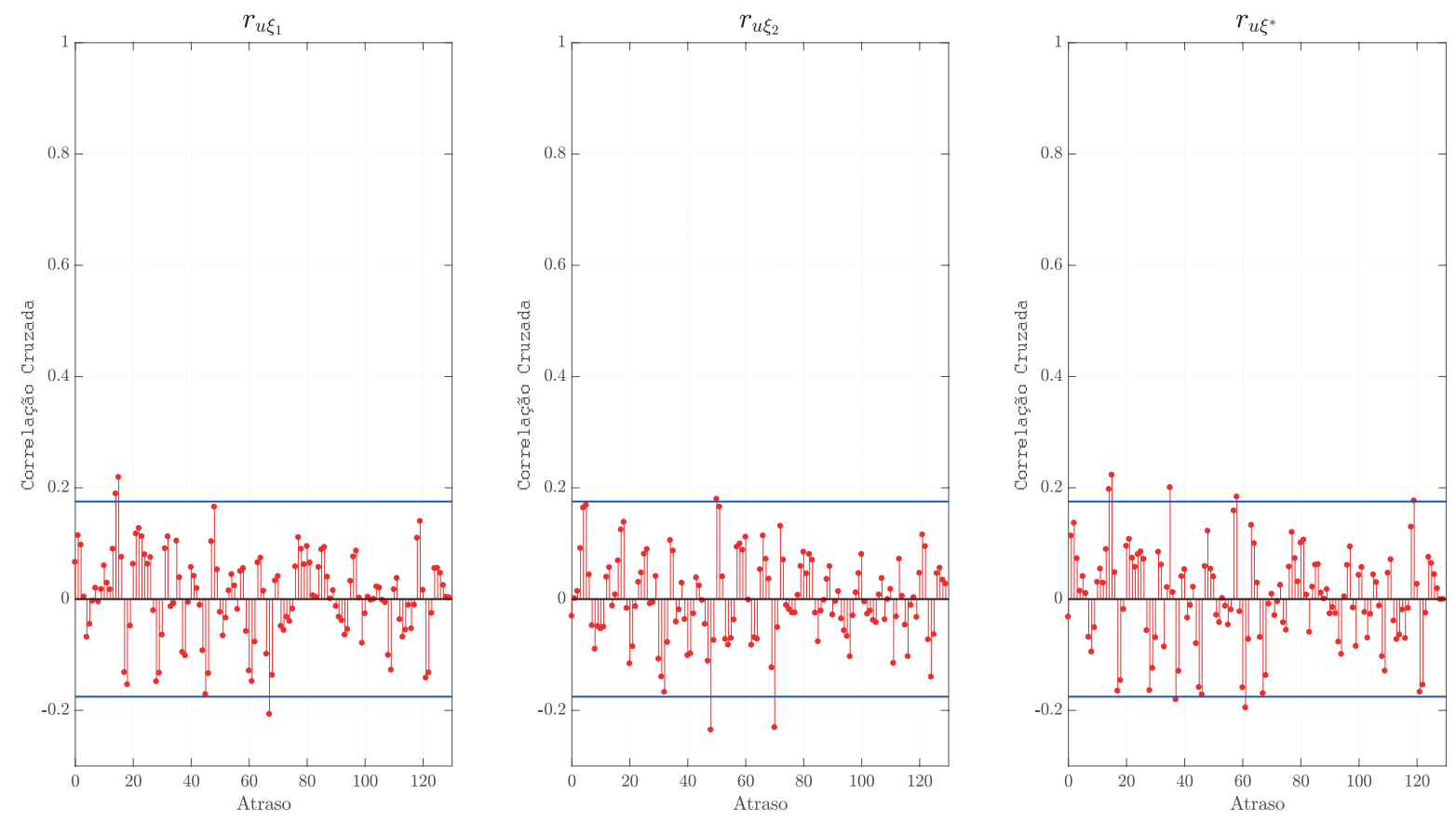

Figura 4. Função de correlação cruzada entre o vetor de resíduos e a entrada, à esquerda para o modelo $y_{1}$, ao centro para o modelo $y_{2}$ e à direita para o modelo $y^{*}$

$$
y=\mu y_{1}+(1-\mu) y_{2} .
$$

As funções $y, y_{1}$ e $y_{2}$ são desconhecidas porém assumindo estabilidade é possível calcular sua área. Integrando os dois 

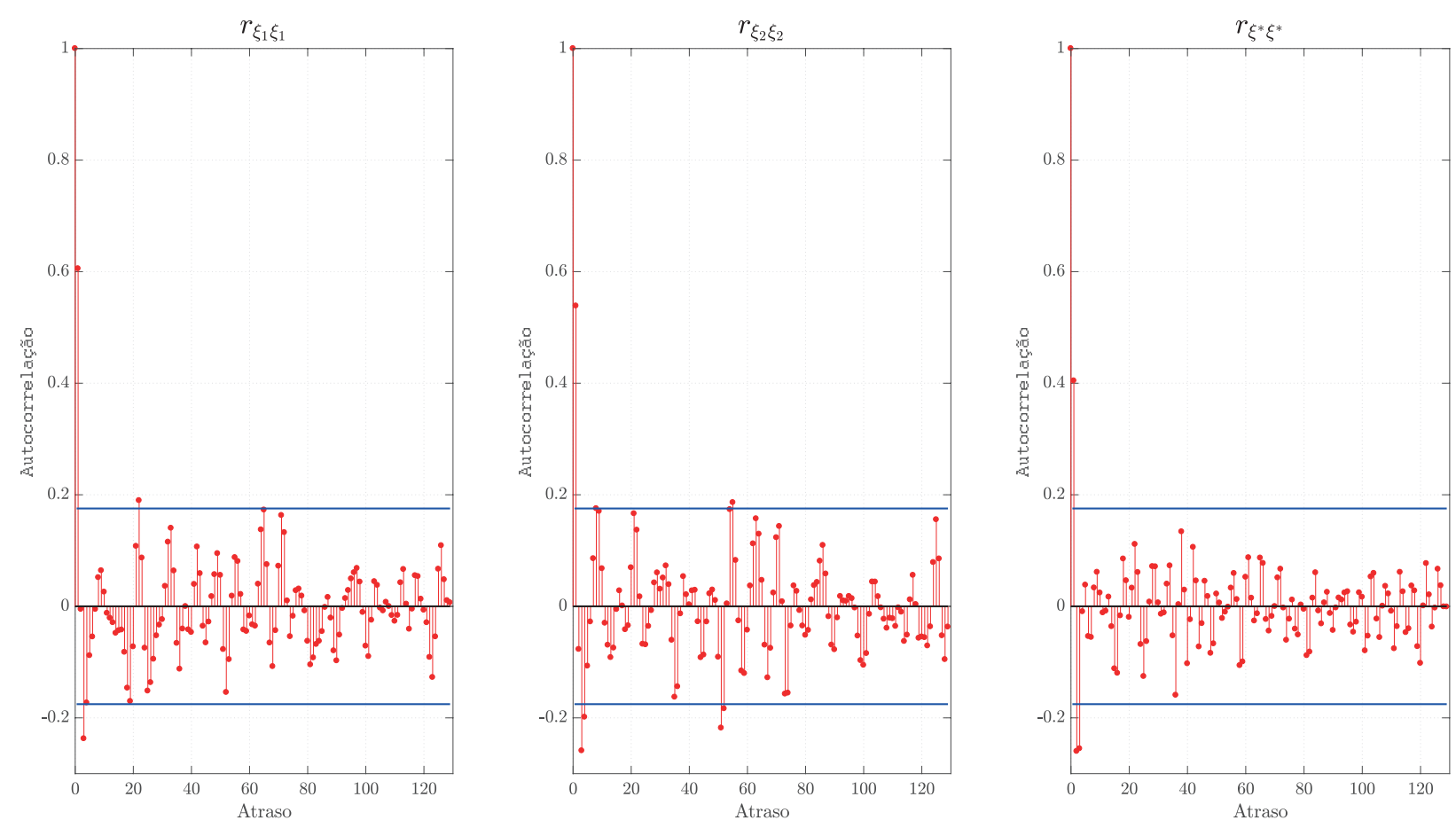

Figura 5. Função de autocorrelação do vetor de resíduos, à esquerda para o modelo $y_{1}$, ao centro para o modelo $y_{2}$ e à direita para o modelo $y^{*}$

lados da Equação (17):

$$
\int_{0}^{n} y d t=\int_{0}^{n} \mu y_{1}+(1-\mu) y_{2} d t .
$$

Reagrupando os termos em (18) pode-se então encontrar uma estimativa do valor $\mu$ que faça com que $y^{*}$ seja aproximadamente $y$ :

$$
\mu=\frac{\int_{0}^{n} y(t) d t-\int_{0}^{n} y_{2}(t) d t}{\int_{0}^{n} y_{1}(t) d t-\int_{0}^{n} y_{2}(t) d t} .
$$

Ou no domínio do tempo discreto:

$$
\mu=\frac{\sum_{k=0}^{n} y_{k}-\sum_{k=0}^{n} y_{2_{k}}}{\sum_{k=0}^{n} y_{1_{k}}-\sum_{k=0}^{n} y_{2_{k}}} .
$$

Um resumo do procedimento pode ser visto no Algoritmo 1. Foram realizadas a análise do NRMSE, a análise de resíduos e a análise do MSE das curvas estáticas para o modelo combinado e para os dois modelos utilizados na interpolação e seus resultados foram comparados.

\section{RESULTADOS}

O procedimento descrito na Seção anterior e resumido no Algoritmo 1 foi implementado utilizando os dados do sistema (7) e os modelos (8) e (9). A simulação livre do modelo combinado bem como dos modelos utilizados na combinação podem ser vistos na parte de cima da Figura 3. Abaixo da Figura 3 podem ser vistas as curvas estáticas para os mesmos modelos.

O modelo interpolado foi validado dinamicamente a partir do índice NRMSE e por meio da análise dos resíduos de simulação livre na qual calculou-se a autocorrelação do vetor de resíduos $\xi$ que pode ser visto na Figura 5 bem

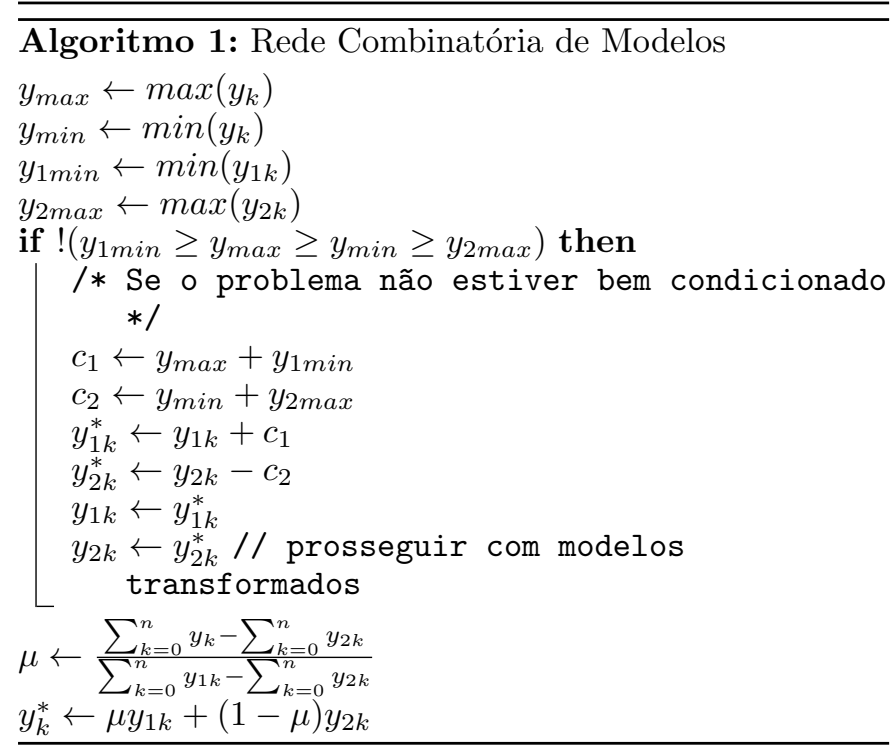

como a correlação cruzada entre o vetor de resíduos $\xi$ e o vetor de entrada $u$ que pode ser visto na Figura 4. Para quantificar esta análise foram calculadas as energias do vetor de resíduos e das funções de autocorrelação e correlação cruzada. Para a validação estática foi calculado o índice MSE das curvas estáticas dos modelos em comparação à curva estática do sistema real. A Tabela I apresenta um comparativo entre o desempenho dos modelos.

Pela Tabela I percebe-se que o modelo combinado obteve uma diminuição de cerca de $65 \%$ no índice NRMSE e uma redução de $95 \%$ no índice estático MSE se comparado com os modelos utilizados em sua obtenção. O modelo combinado obteve também uma redução de $87 \%$ na energia do vetor de resíduos e uma redução de $21 \%$ na energia da 
autocorrelação do vetor de resíduos. É importante frisar que o vetor de resíduos $\xi$ utilizado na análise são os resíduos de predição livre, ou seja, a diferença entre a predição livre dos modelos e os dados reais do sistema.

Tabela I. Comparação de desempenho dos modelos

\begin{tabular}{|l|l|l|l|l|l|}
\hline Modelo & NRMSE & $E_{\xi}$ & $E_{r_{\xi \xi}}$ & $E_{r_{\xi u}}$ & $M S E$ \\
\hline$y_{1}$ & 0,3071 & 1,8008 & 2,0916 & 1,4353 & 0,0268 \\
\hline$y_{2}$ & 1,2949 & 26,829 & 2,1868 & 1,6116 & 0,3188 \\
\hline$y^{*}$ & 0,1086 & 0,2361 & 1,6652 & 1,4701 & 0,0012 \\
\hline
\end{tabular}

É possível notar também que, mesmo os submodelos utilizados possuindo estruturas diferentes daquela pertencente ao sistema a ser identificado, ou seja, os regressores dos submodelos diferem do espaço de regressores do sistema original, o modelo composto obteve uma boa representatividade do sistema, alcançando uma melhora significativa nos índices de validação dinâmicos e estático analisados.

\section{CONCLUSÕES}

Este trabalho integrante de uma série de 2 artigos, apresentou um método de combinação de modelos que consiste na soma ponderada de submodelos e aplicou a técnica desenvolvida no contexto da identificação multiobjetivo de sistemas. Uma formulação matemática para o cálculo dos pesos foi apresentada e os resultados experimentais mostraram que o modelo interpolado possui um melhor desempenho do que os submodelos utilizados nos índices de validação avaliados.

Como trabalho futuro pretende-se utilizar a abordagem apresentada em outros contextos da modelagem de sistemas, atestando que com dois modelos de baixo desempenho é possível chegar em um modelo de alto desempenho. Pretende-se também expandir a formulação matemática para o cálculo dos pesos para um número arbitrário de submodelos.

\section{AGRADECIMENTOS}

O presente trabalho foi realizado com apoio da Coordenação de Aperfeiçoamento de Pessoal de Nível Superior - Brasil (CAPES) - Código de Financiamento 001. Os autores também agradecem à FAPEMIG, CNPq, e à Universidade Federal de São João del Rei pelo apoio.

\section{REFERÊNCIAS}

Aguirre, L. (2015). Introdução à Identificação de Sistemas - Técnicas Lineares e Não-Lineares Aplicadas a Sistemas Reais. Editora UFMG.

Aguirre, L.A., Furtado, E.C., e Tôrres, L.A. (2006). Evaluation of dynamical models: Dissipative synchronization and other techniques. Physical Review E - Statistical, Nonlinear, and Soft Matter Physics, 74(6), 1-16.

Barbosa, A.M. (2010). Técnicas de otimização bi-objetivo para a determinação da estrutura de modelos NARX. Dissertação de Mestrado, Programa de Pós Graduação em Engenharia Elétrica, Universidade Federal de Minas Gerais, Belo Horizonte, Brasil, 3189-3196.
Barroso, M.F.S., Takahashi, R.H.C., e Aguirre, L.A. (2007). Multi-objective parameter estimation via minimal correlation criterion. Journal of Process Control, 17(4), 321-332.

Beykal, B., Boukouvala, F., Floudas, C.A., e Pistikopoulos, E.N. (2018). Optimal design of energy systems using constrained grey-box multi-objective optimization. Computers and Chemical Engineering, 0, 1-15.

Chen, S. e Billings, S.A. (1989). Representations of nonlinear systems: The NARMAX model. International Journal of Control, 49(3), 1013-1032.

Chevtchenko, S.F., Vale, R.F., e Macario, V. (2018). Multi-objective optimization for hand posture recognition. Expert Systems with Applications, 92, 170-181.

Gao, J., He, Q., Gao, H., Zhan, Z., e Wu, Z. (2018). Design of an efficient multi-objective recognition approach for 8-ball billiards vision system. Kuwait Journal of Science, 45(1), 39-53.

Johansen, T.A. (2000). Multi-Objective Identification Of Fir Models. 10-10.

Leontaritis, I.J. e Billings, S.A. (1985a). Input-output parametric models for non-linear systems Part I: Deterministic non-linear systems. International Journal of Control, 41(2), 303-328.

Leontaritis, I.J. e Billings, S.A. (1985b). Input-output parametric models for nonlinear systems, part II: stochastic nonlinear system. International Journal of Control, 41(4), 329-344.

Martins, S.A.M., Nepomuceno, E.G., e Barroso, M.F.S. (2013). Improved structure detection for polynomial NARX models using a multiobjective error reduction ratio. Journal of Control, Automation and Electrical Systems, 24(6), 764-772.

Nepomuceno, E.G., Takahashi, R.H., e Aguirre, L.A. (2007). Multiobjective parameter estimation for nonlinear systems: Affine information and least-squares formulation. International Journal of Control, 80(6), 863871.

Nepomuceno, E.G., Takahashi, R.H.C., Amaral, G.F., e Aguirre, L.A. (2003). Nonlinear Identification Using Prior Knowledge of Fixed Points: a Multiobjective Approach. International Journal of Bifurcation and Chaos, 13(05), 1229-1246.

Ok, S.y., Jung, S., e Song, J. (2018). Multiobjective Optimization Approach for Robust Bridge Damage Identification against Sensor Noise. 2018.

Piroddi, L. e Spinelli, W. (2003). An identification algorithm for polynomial NARX models based on simulation error minimization. International Journal of Control, 76(17), 1767-1781.

Ramalho, F.D., Ekel, P.Y., Pedrycz, W., Júnior, J.G.P., e Soares, G.L. (2019). Multicriteria decision making under conditions of uncertainty in application to multiobjective allocation of resources. Information Fusion, 49, 249 $-261$.

Vignoni, A., Bajur, A., Knust, E., e Sbalzarini, I.F. (2018). Multi-objective identification from fluorescence recovery after photobleaching experiments: Understanding morphogenetic regulation of recovery after photobleaching experiments: Understanding morphogenetic regulation of epithelial polarity. IFAC-PapersOnLine, 51(19), 8-11. 